\section{Regulation of the TSC pathway by LKB1: evidence of a molecular link between tuberous sclerosis complex and Peutz-Jeghers syndrome}

\author{
Michael N. Corradetti, ${ }^{1,2,5}$ Ken Inoki, ${ }^{1,2,5,6}$ \\ Nabeel Bardeesy, ${ }^{4}$ Ronald A. DePinho, ${ }^{4}$ and \\ Kun-Liang Guan 1,2,3,7 \\ ${ }^{1}$ Life Sciences Institute, ${ }^{2}$ Department of Biological Chemistry, \\ and ${ }^{3}$ Institute of Gerontology, University of Michigan, Ann \\ Arbor, Michigan 48109, USA; ${ }^{4}$ Department of Adult \\ Oncology, Dana-Farber Cancer Institute, and Departments of \\ Medicine and Genetics, Harvard Medical School, \\ Boston, Massachusetts 02115, USA
}

Tuberous sclerosis complex (TSC) and Peutz-Jeghers syndrome (PJS) are dominantly inherited benign tumor syndromes that share striking histopathological similarities. Here we show that LKB1, the gene mutated in PJS, acts as a tumor suppressor by activating TSC2, the gene mutated in TSC. Like TSC2, LKB1 inhibits the phosphorylation of the key translational regulators S6K and 4EBP1. Furthermore, we show that LKB1 activates TSC2 through the AMP-dependent protein kinase (AMPK), indicating that LKB1 plays a role in cell growth regulation in response to cellular energy levels. Our results suggest that PJS and other benign tumor syndromes could be caused by dysregulation of the TSC2/mTOR pathway.

Supplemental material is available at http://www.genesdev.org.

Received February 29, 2004; revised version accepted April 27, 2004.

Tuberous sclerosis complex (TSC) is an autosomal dominant syndrome characterized by the development of benign tumors termed hamartomas in a wide range of tissues. The majority of TSC cases are caused by mutation in either the tsc1 or tsc2 tumor suppressor genes (Young and Povey 1998). The proteins TSC1 and TSC2 form a functional complex and inhibit cell growth by negatively regulating the mammalian target of rapamycin (mTOR; Kwiatkowski 2003). mTOR controls cell growth by phosphorylating key substrates such as p70 ribosomal S6 kinase 1 (S6K) and eukaryote initiation factor 4E binding protein 1 (4EBP1; Jacinto and Hall 2003). Peutz-Jeghers syndrome (PJS), another dominantly inherited genetic disorder, is characterized by the formation of gastroin-

[Keywords: LKB1; TSC2; Peutz-Jeghers syndrome; tuberous sclerosis complex; mTOR; AMPK]

${ }^{5}$ These authors contributed equally to this work. Corresponding authors.

6E-MAIL inokik@umich.edu; FAX (734) 647-9702.

7E-MAIL kunliang@umich.edu; FAX (734) 647-9702.

Article and publication are at http://www.genesdev.org/cgi/doi/10.1101/ gad.1199104 testinal hamartomas that happen to be histologically similar to those observed in TSC patients (Devroede et al. 1988). PJS is associated with mutations in the $1 \mathrm{~kb} 1$ tumor suppressor gene, which codes for a serine/threonine kinase. Although extensive work has been performed on the molecular pathogenesis of TSC, the molecular mechanism of LKB1 as a tumor suppressor has remained elusive.

Recent studies from several laboratories have demonstrated that LKB1 phosphorylates and activates AMPK, representing the first convincing physiological target of LKB1 (Hawley et al. 2003; Hong et al. 2003; Woods et al. 2003, Shaw et al. 2004). AMPK is a multimeric protein, and its kinase activity is enhanced by both phosphorylation and high intracellular AMP levels. The amount of AMP in the cell is inversely proportional to the amount of ATP, and high levels of AMP are present under low energy conditions. Under such conditions, AMPK is activated and phosphorylates numerous substrates to suppress anabolism and enhance catabolism, thereby regulating cellular energy homeostasis (Hardie and Hawley 2001). LKB1 potentiates the effect of AMP on AMPK by phosphorylating AMPK on Thr 172, a residue found in the AMPK activation loop.

Previous studies in our laboratory have demonstrated that activated AMPK phosphorylates and activates the TSC2 tumor suppressor protein (Inoki et al. 2003). This AMPK-dependent regulation of TSC2 is especially important for cellular energy response because cells expressing TSC2 mutants that cannot be phosphorylated by AMPK undergo apoptosis under energy starvation conditions (Inoki et al. 2003). TSC2 displays GTPase activating protein (GAP) activity toward the small G-protein Rheb (Ras homolog enriched in brain), and both biochemical and genetic studies have established that TSC2 acts through Rheb to inhibit mTOR function (Shamji et al. 2003). Recent studies have also found that mTOR activation is important for the secretion of vascular endothelial growth factor (VEGF), which is a potent prooncogenic factor that increases the growth of blood vessels near tumors (Treins et al. 2002). Consistent with this observation, VEGF secretion is enhanced in $\mathrm{TSC} 2^{-/-}$ cells, and rapamycin effectively inhibits the secretion of VEGF from these cells (Brugarolas et al. 2003; El-Hashemite et al. 2003). $\mathrm{LKB}^{-/-}$cells are also reported to secrete high levels of VEGF compared with their cognate wild-type cells (Ylikorkala et al. 2001; Bardeesy et al. 2002). Given the biochemical relationship between LKB1 and AMPK, the observation that $\mathrm{LKB1}^{-1-}$ and $\mathrm{TSC}^{-/-}$cells display high VEGF expression, and the histological similarity between the hamartomas of PJS and TSC, we examined the functional relationship between the two tumor suppressors, LKB1 and TSC2.

\section{Results and Discussion}

The best-characterized cellular function of TSC2 is to inhibit the phosphorylation of the translational regulators S6K and 4EBP1 (Goncharova et al. 2002; Inoki et al. 2002; Kwiatkowski et al. 2002). Therefore, we examined the effect of LKB1 overexpression on the phosphorylation of S6K and 4EBP1 in HEK293 cells. Our data show that coexpression of LKB1 significantly decreases the phosphorylation of coexpressed S6K (Fig. 1A). Similarly, 
A

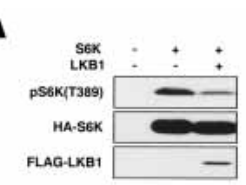

B

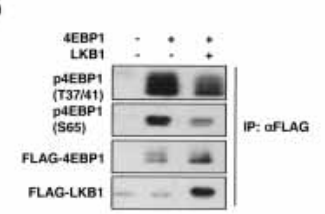

C
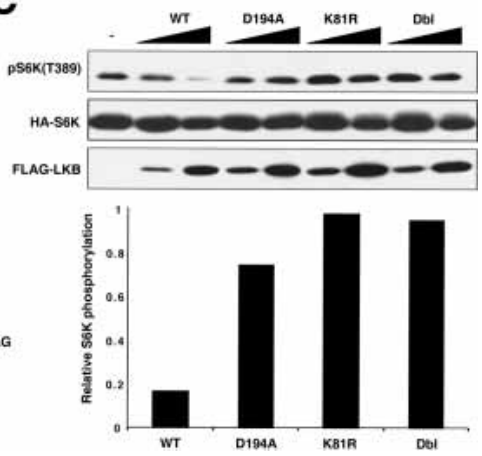

Figure 1. LKB1 inhibits the phosphorylation of S6K and 4EBP1. (A) LKB1 decreases the activation status of coexpressed S6K. HA-S6K and Flag-LKB1 were cotransfected into HEK293 cells, and the phosphorylation of S6K was monitored with a phospho-specific antibody for the S6K activation site, T389. The level of HA-S6K in cell lysates was determined by immunoblotting with an anti-HA antibody, and LKB1 expression was monitored with an anti-Flag antibody. $(B)$ LKB1 decreases the phosphorylation of 4EBP1. Flag-4EBP1 and FlagLKB1 were cotransfected into HEK293 cells, and Flag antibody was used to immunoprecipitate (IP) 4EBP1 and LKB1. The activation state of 4EBP1 was monitored with phospho-specific antibodies against S65 and T37/41. Levels of 4EBP1 and LKB1 were monitored via an anti-Flag immunoblot (IB). (C) LKB1 kinase activity is required for full inhibition of S6K. HA-S6K was cotransfected into HEK293 cells with either wild-type LKB1 or one of three kinase inactive mutants: D194A, a catalytic mutant; K81R, an ATP loading mutant; and $\mathrm{Dbl}$, a double mutant incorporating both mutations. Kinase inactive mutants of LKB1 were unable to significantly inhibit phosphorylation of S6K on T389. Quantitation was performed on the sample with higher expression for each construct.

LKB1 also inhibits the phosphorylation of coexpressed 4EBP1 (Fig. 1B). We also examined the effect of kinase inactive mutants on the phosphorylation of coexpressed S6K. Three kinase inactive LKB1 mutants were tested: K81R, an ATP-binding pocket mutant; D194A, a catalytic mutant; and $\mathrm{Dbl}$, a double mutant incorporating both mutations. Our data clearly show that the kinase activity of LKB1 is required for its ability to inhibit the phosphorylation of S6K (Fig. 1C). The above experiments support the hypothesis that LKB1 inhibits cell growth by decreasing the phosphorylation of S6K and 4EBP1 and are consistent with the notion that LKB1 positively regulates TSC2 function.

To further support the physiological relevance of LKB1 in S6K regulation, we used RNA interference to downregulate the endogenous LKB1 protein. Two different short interfering RNA oligonucleotides were tested: LKBliSP, a pool of several mixed siRNAs; and LKBlicust, a single siRNA. Consistent with the overexpression experiments, down-regulation of LKB1 by RNA interference reproducibly causes a mild increase of endogenous S6K phosphorylation in HEK293 cells at all concentrations of RNAi tested (Fig. 2A). These results further support an inhibitory role of LKB1 in S6K phosphorylation.

It has recently been demonstrated that AMPK is a physiological substrate of LKB1 (Hawley et al. 2003; Hong et al. 2003; Woods et al. 2003; Shaw et al. 2004). We also observed that expression of LKB1 causes an increase of AMPK phosphorylation in the activation loop (T172) of both cotransfected and endogenous AMPK (Supplementary Fig. 1A,B; Hardie and Hawley 2001). Furthermore, the activation of AMPK by LKB1 is supported by the observation that overexpressed LKB1 increases the phosphorylation of acetyl CoA-carboxylase (ACC), a physiological substrate of AMPK (Supplementary Fig. 1B). Moreover, down-regulation of LKB1 by LKBiSP causes a decrease in AMPK phosphorylation; whereas a negative control siRNA directed against EGFP has no effect on phosphorylation of AMPK (Supplementary Fig. 1C). ATP depletion-mediated phosphorylation of AMPK using 2-deoxyglucose (2-DG) was also compromised by LKB1 RNAi (Supplementary Fig. 1C).

We have previously reported that AMPK activation by cellular energy starvation inhibits S6K phosphorylation (Inoki et al. 2003). To determine the functional significance of AMPK in the regulation of S6K by LKB1, a pharmacological inhibitor of AMPK was tested (Zhou et al. 2001). Treatment with the AMPK inhibitor induces an increase of S6K phosphorylation. More importantly, in the presence of AMPK inhibitor, the inhibitory effect of LKB1 on S6K phosphorylation is blocked (Fig. 2B). To rule out the possibility that the AMPK inhibitor nonspecifically inhibits LKB1, we determined whether the inhibitor caused a measurable decrease in LKB1 autophos-

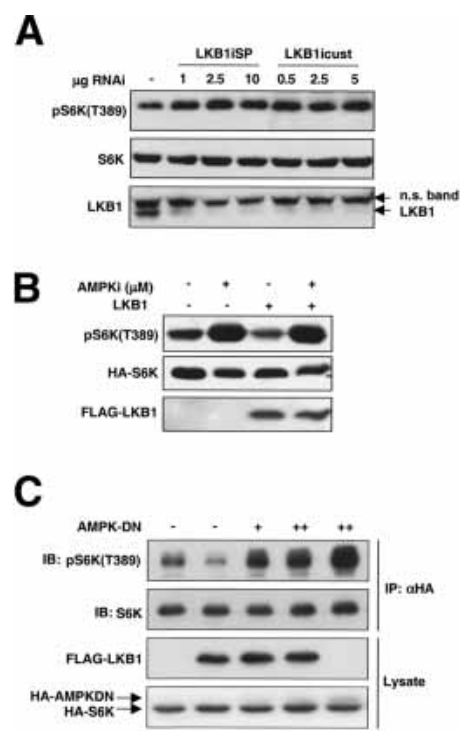

Figure 2. LKB1 functions through AMPK to inhibit S6K. (A) Downregulation of LKB1 with siRNA causes an increase in endogenous S6K activation. LKB1 siRNAs were transfected into HEK293 cells, and the activation status of endogenous S6K was monitored. Two oligonucleotides were tested: LKBliSP is a pool of several siRNAs, and LKBlicust is a single, custom-designed oligonucleotide. Both LKBlicust and LKBliSP were able to cause a decrease in the endogenous LKB1 protein and an increase in S6K activation on T389. Expression levels of LKB1 and S6K were monitored by Western analysis with the indicated antibodies. The high concentration of LKB1 antibody used in this figure (1:5000) detects a nonspecific band (n.s. band) just above the LKB1 protein (as previously described in Bardeesy et al. 2002). (B) An AMPK inhibitor blocks the inhibitory effect of LKB1 on S6K. Flag-LKB1 and HA-S6K were cotransfected into HEK293 cells and treated with $40 \mu \mathrm{M}$ compound C, an AMPK inhibitor (AMPKi), as indicated. Immunoblots were performed with the indicated antibodies. (C) An AMPK dominant-negative construct blocks the inhibitory effect of LKB1 on S6K. HA-S6K expressed in HEK293 cells was immunoprecipitated by anti-HA antibody, and the activation status of S6K was monitored with phosphoS6K antibody. The levels of S6K in immunoprecipitants were monitored by anti-S6K antibody. The levels of Flag-LKB1, HA-S6K, and HA-AMPK-DN (which is a weak band just above HA-S6K) in lysates are also shown. 
phorylation, one readout of LKB1 activity. In vitro kinase assays showed that the AMPK inhibitor had no effect on LKB1 autophosphorylation when added to either intact cells or the kinase buffer (Supplementary Fig. 2A,B), whereas the inhibitor significantly decreased the ability of AMPK to phosphorylate a recombinant fragment of TSC2 (Supplementary Fig. 2C). Consistently, LKB1-induced inhibition of S6K is largely blocked in the presence of a dominant-negative construct of AMPK (Fig. 2C). Taken together, these data are consistent with a model that AMPK functions downstream of LKB1 to inhibit S6K phosphorylation.

It has been reported that HeLa cells contain an undetectable level of endogenous LKB1 (Hawley et al. 2003). To test the effect of lower LKB1 expression levels, we expressed LKB1 in HeLa cells via retroviral infection and found that LKB1 also inhibits S6K in this system (Fig. 3A). As expected, expression of LKB1 increases phosphorylation of AMPK in HeLa cells but has little effect on the phosphorylation of AKT, another key regulator of the TSC pathway (Fig. 3A; Inoki et al. 2002; Manning et al. 2002; Potter et al. 2002). These results are consistent with the notion that it is AMPK but not AKT that mediates the effect of LKB1 on S6K. We performed similar experiments with $\mathrm{LKB1}^{-/-}$MEF cells. Retroviral re-expression of LKB1 increases phosphorylation of AMPK and concomitantly decreases the phosphorylation of S6K in this system as well (Fig. 3A).
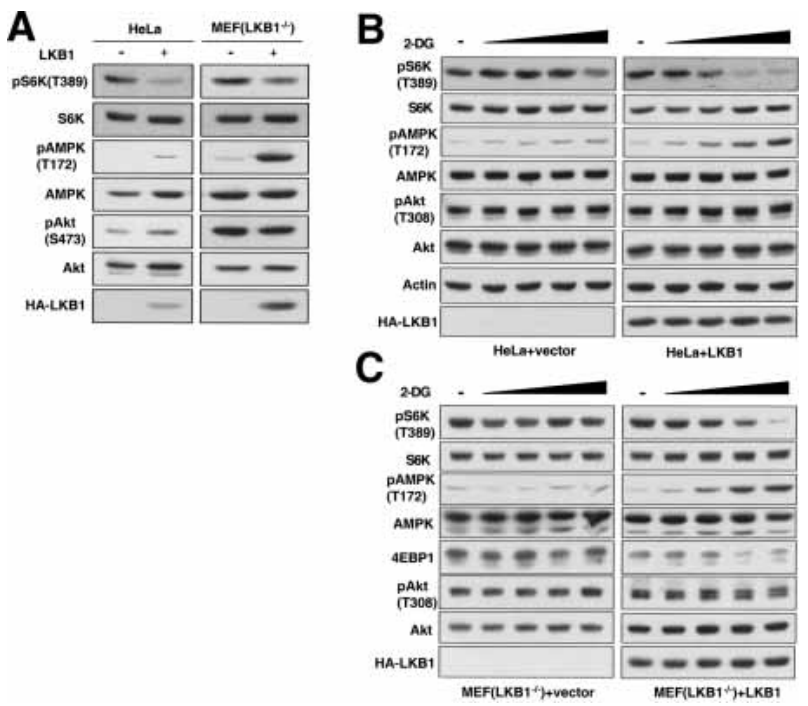

Figure 3. LKB1 inhibits the phosphorylation of S6K in HeLa cells and LKB1 ${ }^{-/-}$MEFs. (A) LKB1 inhibits S6K phosphorylation in HeLa cells and $\mathrm{LKB1}^{-1-}$ MEFs under basal conditions. HeLa cells and $\mathrm{LKB}^{-/-}$MEFs were infected with either HA-LKB1 or empty vectorcontaining retroviruses. Phosphorylation of endogenous proteins was monitored with the indicated phospho-specific antibodies. LKB1 expression was confirmed by HA-antibody. $(B, C)$ LKB1 enhances ATP depletion-induced S6K dephosphorylation. Vector or HA-LKB1-expressing HeLa cells $(B)$ and LKB1 ${ }^{-/-}$MEF cells $(C)$ were treated with various concentrations $(1-25 \mathrm{mM})$ of 2-DG for $10 \mathrm{~min}$. Phosphorylation of endogenous proteins was determined by the indicated phospho-specific antibodies. LKB1 expression was monitored with anti-HA antibody. The exposures selected in $B$ and $C$ were chosen to best illustrate the energy depletion effect on S6K, AMPK, and 4EBP1 phosphorylation; therefore, the Western blots of LKB1-expressing cells were exposed longer than the LKB1-null cells to compare similar basal signal levels.
We also tested the physiological significance of LKB1 in cellular energy response by comparing HeLa cells that express either LKB1 or an empty vector. 2-Dexoyglucose (2-DG), a nonhydrolyzable glucose analog, can inhibit glucose metabolism, thereby causing cellular energy starvation (e.g., see Inoki et al. 2003). HeLa cells without LKB1 expression show little response to 2-DG treatment as determined by the phosphorylation of AMPK and S6K. In contrast, 2-DG induced a significant increase in AMPK phosphorylation in cells that express LKB1 (Fig. 3B). Interestingly, LKB1-expressing cells responded to 2-DG treatment with a dramatic decrease in S6K phosphorylation; however, this effect was absent in the vector control cells. Phosphorylation of AKT was not significantly affected by either LKB1 expression or 2-DG treatment. These data strongly suggest that LKB1 plays an important role in cellular energy response by activating AMPK, thereby inhibiting S6K. We performed similar experiments with $\mathrm{LKB1}^{-{ }^{-}}$MEF cells and obtained results consistent with $\mathrm{LKB} 1$ playing a role in cellular energy response in this system (Fig. 3C). It is worth noting that high concentrations of 2-DG could still induce a reduction in S6K phosphorylation in HeLa cells. This observation could be indicative of the presence of low amounts of LKB1 in the cells or the presence of an AMPK-independent cellular energy response. A possible candidate for an AMPK-independent cellular energy sensor is mTOR, which has a high, $1 \mathrm{mM} \mathrm{K}_{\mathrm{m}}$ for ATP (Dennis et al. 2001). Therefore, mTOR could be functioning as a less sensitive cellular energy sensor under more severe energy starvation conditions.

Our previous studies have established that TSC2 acts directly downstream of AMPK in the cellular energy response pathway, as AMPK directly phosphorylates TSC2 on S1227 and S1345 in response to low energy conditions (Inoki et al. 2003). We found that coexpression of the kinase inactive LKB1-D194A mutant blocks a mobility shift of TSC2 in HEK293 cells (Fig. 4A). Interestingly, LKB1-D194A had little effect on the mobility of TSC2$1227 / 1345 \mathrm{~A}$, a TSC2 mutant in which the AMPK phosphorylation sites have been mutated (Fig. 4B). These results indicate that LKB1 may regulate the phosphorylation of TSC2 via AMPK. To further test this possibility, TSC2 was labeled in vivo with ${ }^{32} \mathrm{P}$-phosphate in the presence or absence of wild-type LKB1 (LKB1-WT), LKB1D194A, and 2-DG. Two-dimensional phosphopeptide mapping shows that LKB1 enhances phosphorylation of TSC2 on AMPK phosphorylation sites (Fig. 4C, panel II), and LKB1-D194A blocks phosphorylation of these sites (Fig. 4C, panel III) in basal conditions. LKB1 or LKB1D194A has little effect on the phosphorylation of the TSC2 2A (TSC 1227/1345A) mutant (data not shown). Furthermore, 2-DG-induced T1227 and S1345 phosphorylation (Fig. 4C, panel V) was significantly reduced by coexpression of LKB1-D194A (Fig. 4C, panel VI). These data support the hypothesis that LKB1 regulates TSC2 phosphorylation by controlling AMPK activity.

To further test the functional relationship between LKB1 and TSC2, TSC2 was down-regulated by RNA interference. Consistent with our model, we observed that the ability of LKB1 to inhibit S6K was significantly blocked when TSC2 protein was down-regulated (Fig. 4D). Similar experiments were performed in $\mathrm{TSC}^{-1-}$ cells. Our data show that LKB1 cannot decrease S6K phosphorylation in TSC2 $2^{-/}$cells, whereas rapamycin is still able to inhibit S6K phosphorylation effectively 


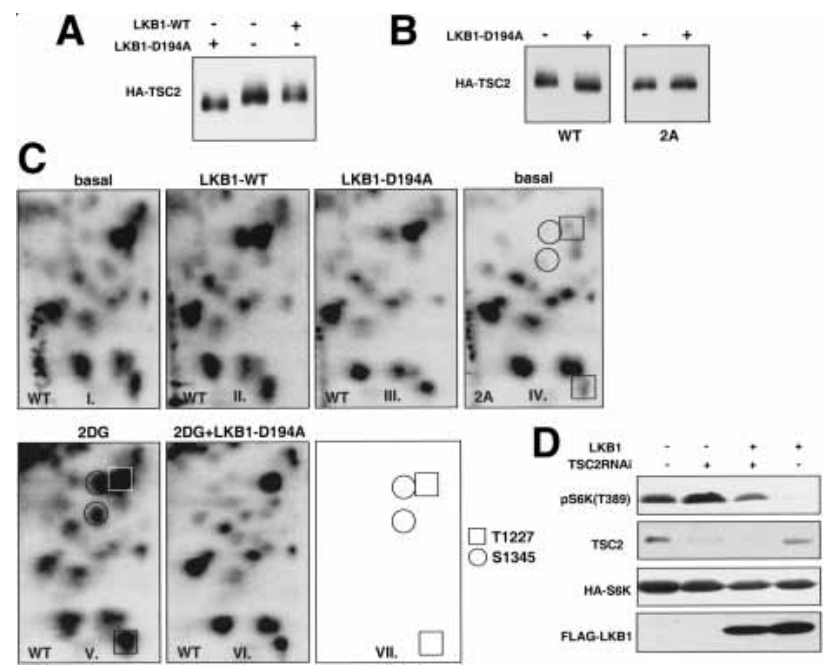

Figure 4. LKB1 functions through TSC2. (A) A kinase inactive LKB1 construct, LKB1-D194A, causes a downshift of cotransfected TSC2. LKB1 or LKB1-D194A was coexpressed in HEK293 cells with HA-TSC2 and Myc-TSC1. Mobility shift assay of HA-TSC2 was performed by running lysates on $6 \%$ SDS-PAGE followed by immunoblotting with anti-HA antibody. (B) LKB1-D194A causes a downshift of coexpressed wild-type HA-TSC2 but not of HA-TSC 2A, a mutant of TSC2 that eliminates the AMPK phosphorylation residues T1227 and S1345. The experimental design is the same as in $A$. (C) LKB1-D194A affects T1227 and S1345 phosphorylation on TSC2. HA-TSC2 and Myc-TSC1 were coexpressed with LKB1 or LKB1D194A in HEK293 cells. In vivo labeling was performed, and immunoprecipitated HA-TSC2 was subjected to two-dimensional phospho-peptide mapping. Phosphorylation of the shaped spots depicted in panel $V$ were enhanced by 2-DG treatment. Phosphorylation of the corresponding spots were decreased by LKB1-D194A (panels $I, V I$ ) and eliminated by (2A) T1227A/S1345A mutations (panel IV). Panel VII represents a schema for indicating AMPK-dependent phospho-peptide changes (also depicted in panels $I V$ and $V$; Inoki et al. 2003). (D) TSC2 is required for LKB1 to fully inhibit S6K. TSC2 was down-regulated in HEK293 cells by RNA interference in the presence or absence of cotransfected LKB1. The activation status of HA-S6K was monitored on T389. The levels of S6K, LKB1, and endogenous TSC2 were monitored via immunoblot with the indicated antibodies.

(Supplementary Fig. 3A). Therefore, the presence of TSC2 protein is required for LKB1 to inhibit phosphorylation of S6K. We also tested the effect of LKB1 on S6K in the presence of overexpressed Rheb, the downstream target of TSC2. The data indicate that LKB1 is unable to repress S6K phosphorylation in the presence of overexpressed wild-type Rheb or a more active mutant of Rheb, Rheb-L64 (Supplementary Fig. 3B). LKB1 is still able to repress $\mathrm{S} 6 \mathrm{~K}$ phosphorylation in the presence of an inactive mutant of Rheb, Rheb-5A (Supplementary Fig. 3B). Taken together, these data are consistent with our model that LKB1 acts upstream of AMPK and TSC2 to inhibit S6K phosphorylation (Fig. 5E).

To determine the physiological relevance of LKB1 in the context of the TSC2/mTOR pathway, we examined the effect of low energy conditions on cell viability in $\mathrm{LKB1}^{-/-}$MEFs. Our laboratory has previously shown that cells null for TSC2 undergo apoptotic cell death when cultured in glucose-free media for $72 \mathrm{~h}$ (Inoki et al. 2003). The results of similar experiments performed in LKB1null cells are consistent with the idea that LKB1 is also required to protect cells from energy deprivation-in- duced apoptosis (Fig. 5A). Moreover, we found that cleavage of caspase-3, a marker of apoptotic cell death, was significantly enhanced in response to glucose depletion in LKB1-null cells but not in cells that express LKB1. This effect was ablated by rapamycin, implying that apoptosis caused by the absence of LKB1 under low energy conditions is mTOR-dependent (Fig. 5B).

In addition to energy depletion-induced apoptosis, the expression and secretion of VEGF is another important marker of hyperactive mTOR signaling (Treins et al. 2002). Augmented VEGF secretion is especially significant in the context of tumorigenesis, because increased blood vessel formation mediated by VEGF allows for the maintenance and growth of tumors beyond sizes normally attainable with no additional blood supply. It has been previously shown that TSC2 $2^{-/-}$cells secrete significantly more VEGF than TSC2 $2^{+/+}$cells, and this secretion is partially inhibited by rapamycin (Brugarolas et al. 2003; El-Hashemite et al. 2003). It has also been shown that $\mathrm{LKB1}^{-/-}$cells secrete significantly higher levels of VEGF than $\mathrm{LKB1}^{+/+}$cells (Ylikorkala et al. 2001; Bardeesy et al. 2002). We were able to recapitulate these results by both ELISA (Fig. 5C) and Western analysis (Fig. 5D); and we extended these data to show that rapamycin significantly inhibits VEGF expression and secretion in cells null for LKB1. Therefore, we propose that the vascular nature of PJS tumors (similar to that of TSC tumors) may be at least partially due to high levels of VEGF caused by dysregulated mTOR signaling.

LKB1 is one of the first protein kinases shown to function as a tumor suppressor (Jenne et al. 1998). Despite extensive biochemical, genetic, and cell biological studies, the molecular mechanism of LKB1 as a tumor suppressor has been elusive. However, the identification of AMPK as a physiological substrate of LKB1 and the observations described in this report provide an important clue of how LKB1 could negatively regulate cell growth. We propose the following mechanism to explain how LKB1 activates the TSC2 tumor suppressor (Fig. 5E): LKB1 directly phosphorylates and activates AMPK. The active AMPK then phosphorylates TSC2 to enhance TSC2 function. TSC2 subsequently inhibits mTOR function via TSC2's GAP activity toward the Rheb small GTPase. Therefore, we postulate that LKB1 negatively regulates cell growth by inhibiting the phosphorylation of important mTOR targets such as S6K and 4EBP1. It should be noted that, in addition to AMPK, LKB1 can phosphorylate and activate several AMPK-related kinases-although these kinases are not regulated by AMP (Lizcano et al. 2004). It remains to be seen whether these AMPK-related kinases can also contribute to LKB1-mediated phosphorylation of TSC2. However, our results make it clear that the LKB1-AMPK kinase cascade likely plays a major role in TSC2-mTOR regulation of cellular energy response. Moreover, our data indicate that mutation in TSC2 or LKB1 produces similar cellular phenotypes (rapamycin-sensitive apoptosis and VEGF production), supporting that the two tumor-suppressor proteins function in the same pathway (Fig. 5E).

It should also be noted that mutations in the phosphoinositide phosphatase PTEN are associated with Cowden's disease and the Bannayan-Riley-Ruvalcaba syndrome, two other dominantly inherited hamartoma syndromes (Eng 2003). Loss of PTEN leads to an increase in 3-phosphoinositide concentration and subsequent activation of $\mathrm{AKT}$, and AKT has been shown to inhibit 


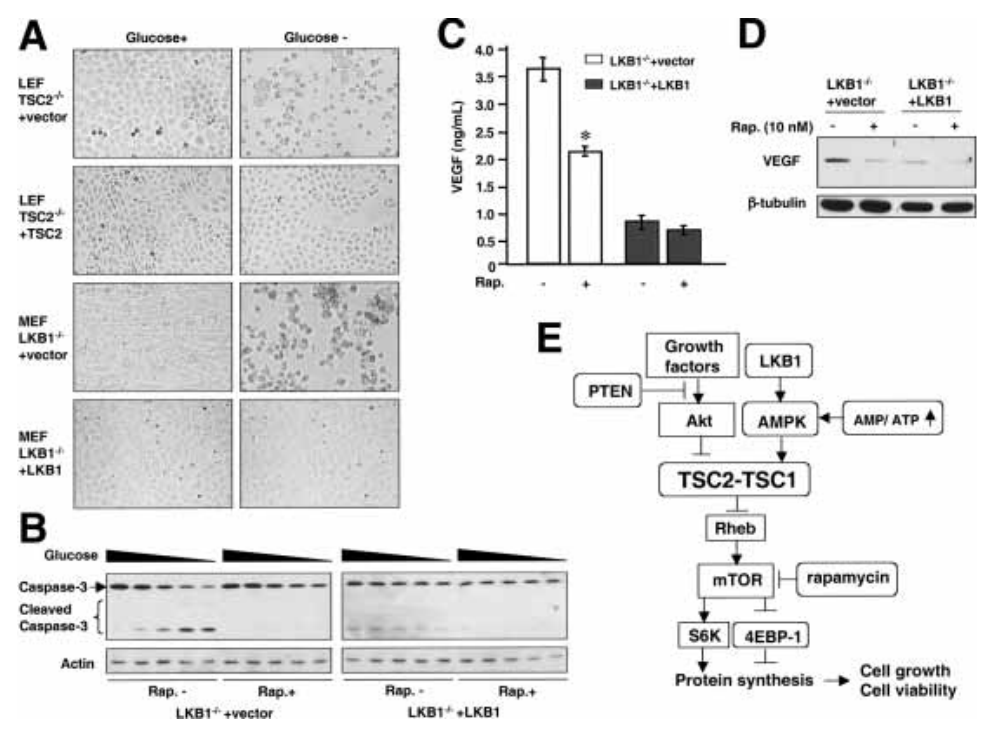

Figure 5. Cells null for TSC2 or LKB1 display similar phenotypes for certain biological responses. $(A)$ TSC2 and LKB1 play essential roles in protecting cells from glucose depletion-induced cell death. LEF cells $\left(\mathrm{TSC}^{-/-}\right)$stably expressing empty vector or TSC2, and MEF cells $\left(\mathrm{LKB}^{-/-}\right)$ stably expressing empty vector or LKB1 were cultured in $25 \mathrm{mM}$ glucose (glucose + ) or glucose-free medium (glucose-). Photos were taken after $72 \mathrm{~h}$ in culture for LEF cells and $48 \mathrm{~h}$ for MEF cells. (B) Rapamycin inhibits glucose depletion-induced caspase-3 activation in $\mathrm{LKB}^{-/}$ MEF cells. MEF $\left(\mathrm{LKB}^{-/-}\right)$cells stably expressing vector or LKB1 were cultured for $30 \mathrm{~h}$ in media containing various concentrations of glucose $(1-25 \mathrm{mM})$ in the presence or absence of rapamycin $(20 \mathrm{nM})$. Immunoblots of caspase-3, cleaved caspase- 3 , and actin are shown. $(C)$ Rapamycin inhibits VEGF secretion in LKB1 ${ }^{-/}$MEF cells. Equal numbers of $\mathrm{LKB}^{-/-} \mathrm{MEF}$ cells stably expressing empty vector or LKB1 were cultured for $36 \mathrm{~h}$ with or without rapamycin. The concentration of secreted VEGF in the medium was determined by ELISA. Data are expressed as a mean, and one standard deviation is indicated by the error bars $(n=3)$. The asterisk indicates that the reduction in VEGF secretion induced by rapamycin in LKB1null cells is significant, as well as the increased expression of VEGF in the LKB1-null cells relative to the reexpressed cells $(p<0.0001)$. (D) Rapamycin inhibits VEGF expression in LKB1 $1^{-/-}$MEF cells. Equal numbers of MEFs stably expressing empty vector or LKB1 were cultured for $36 \mathrm{~h}$ with or without rapamycin. VEGF expression in cell lysates was monitored by VEGF immunoblot, and protein level was monitored by $\beta$-tubulin immunoblot. (E) A proposed model for a role of LKB1 in the TSC pathway. Loss of LKB1 is a hallmark of Peutz-Jeghers syndrome. Less LKB1 would cause a decrease in the activation status of AMPK on T172 and of TSC2 on S1227 and S1335, relieving TSC2's inhibitory effect on Rheb. Higher Rheb activity would subsequently drive mTOR activation. Two key targets of mTOR-S6K and 4EBP1-are shown. We propose a model in which dysregulated increases in the activity S6K, 4EBP1, and other mTOR targets could lead to hamartoma formation in PJS in an analogous way to that of TSC. In a similar way, hamartoma-causing mutations in PTEN affect the activation status of AKT, a molecule that also acts on TSC2. Thus, the TSC2/mTOR pathway could represent a common pathway for hamartoma formation. Pointed arrowheads indicate activation, and flat arrowheads indicate inhibition.

TSC2 function. Thus, the pathophysiology of several syndromes associated with benign tumors may converge on the TSC2 and mTOR pathway. It is also especially significant that mTOR is a key downstream target of LKB1 in the regulation of cell growth, because the immunosuppressant drug rapamycin specifically inhibits mTOR. Recently, murine studies and clinical trials have indicated that rapamycin and other mTOR inhibitors may be potent drugs for the treatment of TSC and other neoplasms (Kenerson et al. 2002; Huang and Houghton 2003). Our studies suggest that rapamycin and mTOR inhibitors may also be potential drugs for the comprehensive treatment of PJS.

\section{Materials and methods}

RNA interference

Short interfering RNA oligonucleotides were purchased from Dharmacon. The target sequence for the negative control EGFP siRNA is 5'AAGACAAUCGGCUGCUCUGAU-3'. The target sequence for the custom-designed LKB1 siRNA (LKBicust) is 5'-AAAGGGAUGCUUGAGU ACGAA-3'. The other LKB1 oligonucleotides tested (LKBiSP) were a "Smart Pool" of several siRNAs selected by Dharmacon. TSC2 RNAi oligonucleotides were described previously (Inoki et al. 2003). Oligonucleotides were transfected into HEK293 cells, and lysates were made $\sim 24$ h posttransfection.

VEGF ELISA

VEGF secretion experiments were performed by seeding equal numbers of empty vector- or LKB1-expressing $\mathrm{LKB1}^{-/-}$MEFs in six-well plates. After cell attachment, the media was replaced and treated with $25 \mathrm{nM}$ rapamycin in methanol or methanol only as a vehicle control. Cell culture supernatants were collected $36 \mathrm{~h}$ later and subjected to ELISA for mouse VEGF according to the manufacturer's instructions (R\&D Systems). Statistical analysis was performed by ANOVA followed by Bonferroni's post hoc comparison between selected groups.

Detailed extra information regarding materials and methods is found in the Supplemental Material.

\section{Acknowledgments}

We thank Tianqing Zhu for technical assistance and the members of the Guan laboratory for a critical reading of the manuscript. This work is supported by grants from the $\mathrm{NIH}$ and the Walther Cancer Institute (K.-L.G). M.N.C. is supported by a fellowship from the Training Program in Organogenesis (5-T32-HD075005).

The publication costs of this article were defrayed in part by payment of page charges. This article must therefore be hereby marked "advertisement" in accordance with 18 USC section 1734 solely to indicate this fact.

\section{References}

Bardeesy, N., Sinha, M., Hezel, A.F., Signoretti, S., Hathaway, N.A. Sharpless, N.E., Loda, M., Carrasco, D.R., and DePinho, R.A. 2002. Loss of the Lkb1 tumour suppressor provokes intestinal polyposis but resistance to transformation. Nature 419: 162-167.

Brugarolas, J.B., Vazquez, F., Reddy, A., Sellers, W.R., and Kaelin Jr., W.G. 2003. TSC2 regulates VEGF through mTOR-dependent and -independent pathways. Cancer Cell 4: 147-158.

Dennis, P.B., Jaeschke, A., Saitoh, M., Fowler, B., Kozma, S.C., and Thomas, G. 2001. Mammalian TOR: A homeostatic ATP sensor. Science 294: 1102-1105.

Devroede, G., Lemieux, B., Masse, S., Lamarche, J., and Herman, P.S. 1988. Colonic hamartomas in tuberous sclerosis. Gastroenterology 94: $182-188$.

El-Hashemite, N., Walker, V., Zhang, H., and Kwiatkowski, D.J. 2003. Loss of Tsc1 or Tsc2 induces vascular endothelial growth factor production through mammalian target of rapamycin. Cancer Res. 63: 5173-5177.

Eng, C. 2003. PTEN: One gene, many syndromes. Hum. Mutat. 22: 183 198.

Goncharova, E.A., Goncharov, D.A., Eszterhas, A., Hunter, D.S., Glassberg, M.K., Yeung, R.S., Walker, C.L., Noonan, D., Kwiatkowski, D.J., Chou, M.M., et al. 2002. Tuberin regulates p70 S6 kinase activation and ribosomal protein S6 phosphorylation. J. Biol. Chem. 277: 30958-30967.

Hardie, D.G. and Hawley, S.A. 2001. AMP-activated protein kinase: The 


\section{Corradetti et al.}

energy charge hypothesis revisited. Bioessays 23: 1112-1119.

Hawley, S.A., Boudeau, J., Reid, J.L., Mustard, K.J., Udd, L., Makela, T.P., Alessi, D.R., and Hardie, D.G. 2003. Complexes between the LKB1 tumor suppressor, STRAD $\alpha / \beta$ and $\mathrm{MO} 25 \alpha / \beta$ are upstream kinases in the AMP-activated protein kinase cascade. J. Biol. 2: 28.

Hong, S.P., Leiper, F.C., Woods, A., Carling, D., and Carlson, M. 2003. Activation of yeast Snfl and mammalian AMP-activated protein kinase by upstream kinases. Proc. Natl. Acad. Sci. 100: 8839-8843.

Huang, S. and Houghton, P.J. 2003. Targeting mTOR signaling for cancer therapy. Curr. Opin. Pharmacol. 3: 371-377.

Inoki, K., Li, Y., Zhu, T., Wu, J., and Guan, K.L. 2002. TSC2 is phosphorylated and inhibited by AKT and suppresses mTOR signalling. Nat. Cell Biol. 4: 648-657.

Inoki, K., Zhu, T., and Guan, K.L. 2003. TSC2 mediates cellular energy response to control cell growth and survival. Cell 115: 577-590.

Jacinto, E. and Hall, M.N. 2003. Tor signalling in bugs, brain and brawn. Nat. Rev. Mol. Cell. Biol. 4: 117-126.

Jenne, D.E., Reimann, H., Nezu, J., Friedel, W., Loff, S., Jeschke, R. Muller, O., Back, W., and Zimmer, M. 1998. Peutz-Jeghers syndrome is caused by mutations in a novel serine threonine kinase. Nat. Genet. 18: $38-43$.

Kenerson, H.L., Aicher, L.D., True, L.D., and Yeung, R.S. 2002. Activated mammalian target of rapamycin pathway in the pathogenesis of tuberous sclerosis complex renal tumors. Cancer Res. 62: 5645-5650.

Kwiatkowski, D.J. 2003. Tuberous sclerosis: From tubers to mTOR. Ann. Hum. Genet. 67: 87-96.

Kwiatkowski, D.J., Zhang, H., Bandura, J.L., Heiberger, K.M., Glogauer, M., el-Hashemite, N., and Onda, H. 2002. A mouse model of TSC1 reveals sex-dependent lethality from liver hemangiomas, and upregulation of p70S6 kinase activity in Tsc1 null cells. Hum. Mol. Genet. 11: 525-534.

Lizcano, J.M., Goransson, O., Toth, R., Deak, M., Morrice, N.A., Boudeau, J., Hawley, S.A., Udd, L., Makela, T.P., Hardie, D.G., et al. 2004. LKB1 is a master kinase that activates 13 kinases of the AMPK subfamily, including MARK/PAR-1. EMBO J. 23: 833-843.

Manning, B.D., Tee, A.R., Logsdon, M.N., Blenis, J., and Cantley, L.C. 2002. Identification of the tuberous sclerosis complex-2 tumor suppressor gene product tuberin as a target of the phosphoinositide 3-kinase/AKT pathway. Mol. Cell 10: 151-162.

Potter, C.J., Pedraza, L.G., and Xu, T. 2002. AKT regulates growth by directly phosphorylating Tsc2. Nat. Cell Biol. 4: 658-665.

Shamji, A.F., Nghiem, P., and Schreiber, S.L. 2003. Integration of growth factor and nutrient signaling: Implications for cancer biology. Mol. Cell 12: 271-280.

Shaw, R.J., Kosmatka, M., Bardeesy, N., Hurley, R.L., Witters, L.A., DePinho, R.A., and Cantley, L.C. 2004. The tumor suppressor LKB1 kinase directly activates AMP-activated kinase and regulates apoptosis in response to energy stress. Proc. Nat1. Acad. Sci. 101: 33293335.

Treins, C., Giorgetti-Peraldi, S., Murdaca, J., Semenza, G.L., and Van Obberghen, E. 2002. Insulin stimulates hypoxia-inducible factor 1 through a phosphatidylinositol 3-kinase/target of rapamycin-dependent signaling pathway. J. Biol. Chem. 277: 27975-27981.

Woods, A., Johnstone, S.R., Dickerson, K., Leiper, F.C., Fryer, L.G., Neumann, D., Schlattner, U., Wallimann, T., Carlson, M., and Carling, D. 2003. LKB1 is the upstream kinase in the AMP-activated protein kinase cascade. Curr. Biol. 13: 2004-2008.

Ylikorkala, A., Rossi, D.J., Korsisaari, N., Luukko, K., Alitalo, K., Henkemeyer, M., and Makela, T.P. 2001. Vascular abnormalities and deregulation of VEGF in Lkb1-deficient mice. Science 293: 1323-1326.

Young, J. and Povey, S. 1998. The genetic basis of tuberous sclerosis. Mol. Med. Today 4: 313-319.

Zhou, G., Myers, R., Li, Y., Chen, Y., Shen, X., Fenyk-Melody, J., Wu, M., Ventre, J., Doebber, T., Fujii, N., et al. 2001. Role of AMP-activated protein kinase in mechanism of metformin action. J. Clin. Invest. 108: $1167-1174$. 


\section{CORRIGENDUM}

Genes \& Development 18: 1533-1538 (2004)

\section{Corrigendum: Regulation of the TSC pathway by LKB1: evidence of a molecular link between tuberous sclerosis complex and Peutz-Jeghers syndrome}

Michael N. Corradetti, Ken Inoki, Nabeel Bardeesy, Ronald A. DePinho, and Kun-Liang Guan

It has recently come to the authors' attention that two panels in Figure 5A were duplicated. The original digital images for this figure could not be located; however, we believe that this duplication was caused by an inadvertent error during figure preparation. To correct this mistake, the lower duplicated panels in Figure 5A have been removed, as shown below. This modification has no effect on the conclusion of the paper, as data in Figure 5B similarly demonstrate that the LKB1 mutant cells were more sensitive to glucose starvation. The authors apologize for this error.

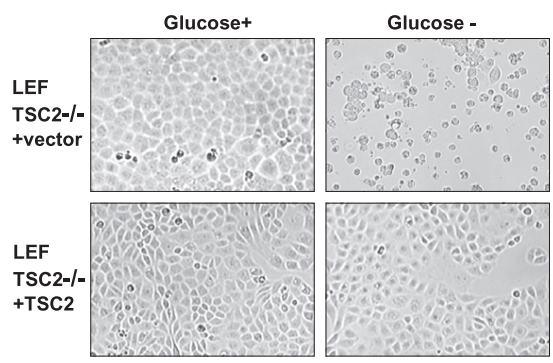

doi:10.1101/gad.324970.119 


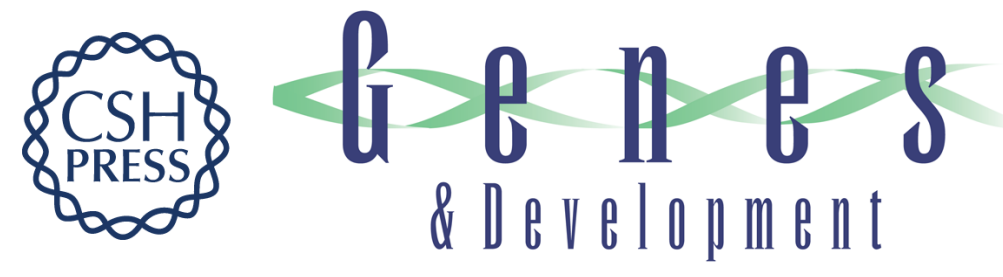

\section{Regulation of the TSC pathway by LKB1: evidence of a molecular link between tuberous sclerosis complex and Peutz-Jeghers syndrome}

Michael N. Corradetti, Ken Inoki, Nabeel Bardeesy, et al.

Genes Dev. 2004, 18:

Access the most recent version at doi:10.1101/gad.1199104

\section{Supplemental http://genesdev.cshlp.org/content/suppl/2004/06/14/18.13.1533.DC1 \\ Material}

Related Content

Corrigendum: Regulation of the TSC pathway by LKB1: evidence of a molecular link between tuberous sclerosis complex and Peutz-Jeghers syndrome

Michael N. Corradetti, Ken Inoki, Nabeel Bardeesy, et al.

Genes Dev. April , 2019 33: 477

References This article cites 28 articles, 9 of which can be accessed free at:

http://genesdev.cshlp.org/content/18/13/1533.full.html\#ref-list-1

Articles cited in:

http://genesdev.cshlp.org/content/18/13/1533.full.html\#related-urls

\section{License}

Email Alerting

Service

Receive free email alerts when new articles cite this article - sign up in the box at the top right corner of the article or click here.

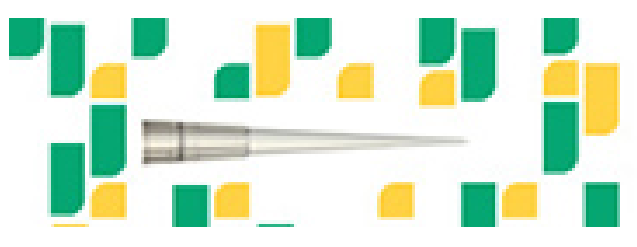

Focused on your science. 